\title{
Surgical Management of Intracerebral Hemorrhage
}

\author{
Arvin R. Wali, Kevin Porras, Peter Abraham, \\ Michael G. Brandel, David Santiago Dieppa, \\ Jeffrey Steinberg, Scott Pannell and \\ Alexander A. Khalessi \\ Additional information is available at the end of the chapter
}

http://dx.doi.org/10.5772/67633

\begin{abstract}
Intracerebral hemorrhage (ICH), defined as bleeding within the brain parenchyma, remains a challenging and controversial neurosurgical entity to treat. ICH has a broad range of etiology - stemming from complications associated with traumatic head injury to complications of hemorrhagic stroke. The role of medical management lies in optimizing blood pressure and intracerebral pressure, preventing secondary injury from complications of the hematoma such as seizures, and correcting coagulopathy. Given the mass effect of a hematoma and the possibility of expansion, surgical interventions attempt to evacuate the clot to restore normal intracerebral pressure and prevent worsening neurologic injury. This chapter reviews the recent controversy associated with surgical evacuation of intracerebral hemorrhage placing particular emphasis on the size and location of the hemorrhage and the methods used to evacuate the expanding ICH. Moreover, this chapter reviews considerations and therapeutic goals of the preoperative and postoperative window to minimize complications and optimize patient care.
\end{abstract}

Keywords: intracerebral hemorrhage, hemorrhagic stroke, surgical intracerebral hemorrhage, neurosurgical interventions

\section{Introduction}

Intracerebral hemorrhage $(\mathrm{ICH})$ is defined as bleeding that takes place within the brain parenchyma and impacts 63,000 people annually within the United States [1, 2]. ICH is a major complication of traumatic brain injury and contributes to $15 \%$ of strokes [3]. Many etiologies are implicated in $\mathrm{ICH}$, including hypertension, vascular anomalies, amyloid angiopathy, 
coagulopathy, brain malignancies, pregnancies, and substance abuse. Depending on the extent of hemorrhage and additional risk factors, ICH prognosis can be highly variable. Extensive hemorrhage has been associated with greater than $30 \%$ mortality rate within one month of injury with only $30 \%$ of patients returning to independent functioning by 6 months [4].

Medical management aims to optimize blood pressure, intracerebral pressure (ICP), coagulopathy, seizure control, fever control, and deep vein thrombosis prophylaxis. Additionally, surgical management aims to prevent neurologic decline through surgical decompression of the skull and evacuation of expanding hematoma [5]. However, the role of surgery in management for ICH remains controversial, and numerous trials have been conducted examining the indications and efficacy of prompt surgical intervention. This chapter will explore the current scientific literature regarding the indications and efficacy of neurosurgical intervention in the management of patients with $\mathrm{ICH}$.

\section{Preoperative diagnostics and workup}

ICH presents with acute-onset focal neurologic deficits, headache (approximately $40 \%$ of patients), elevated systolic blood pressure (50\%), nausea, and vomiting (50\%) [5-7]. Common deficits include, in order of decreasing incidence, paresis, sensory deficits, speech deficits, vision changes, and gait disturbances [6]. Symptoms are often progressive due to hematoma expansion, which tends to occur within the first several hours following $\mathrm{ICH}$. However, patients without hemorrhage growth may still experience substantial neurological deterioration within the first 24 hours [8]. Electrographic seizures may occur in up to a third of patients with $\mathrm{ICH}$, although only half of these are clinically significant [9]. Seizures are more likely to occur with cortical involvement of $\mathrm{ICH}$ and may be associated with neurological deterioration and increased midline shift [10,11]. Patients commonly have altered mental status, and those with large or growing hematomas may experience rapid worsening in Glasgow Coma Scale (GCS) [12].

Non-contrast computed tomography (CT) scan is considered to be the "gold standard" for diagnosis of new $\mathrm{ICH}$ and detection of other forms of intracranial bleeding that may present concomitantly [13]. Magnetic resonance imaging (MRI) may also be beneficial in identifying microhemorrhages, and CT angiogram is sensitive in identifying secondary causes of intraparenchymal bleeding such as aneurysm rupture, vasculitis, intracranial malignancy, or arteriovenous malformations $[14,15]$.

In an emergency setting, ensuring adequate airway, breathing, and cerebral perfusion is essential. For patients with a GCS $<8$, transtentorial herniation, intraventricular hemorrhage, or hydrocephalus, an ICP monitor should be placed to dynamically monitor cerebral perfusion pressure [16]. Management of elevated ICPs in the acute setting includes elevating the head of bed to $30^{\circ}$, sedation, intubation, and hyperventilation of the patient to a $\mathrm{PaCO}_{2}$ of 25-30 and rapidly infusing hypertonic saline or mannitol while preparing the operating room for surgical decompression or clot evacuation $[9,17]$. Management of hemorrhagic mass effect and acute hydrocephalus via external ventricular drain is beneficial for ICP measurement and cerebrospinal fluid (CSF) diversion in these patients and has been shown to significantly reduce mortality in the setting of intraventricular hemorrhage [18, 19]. 


\section{Surgical management}

The role of surgery in the treatment of intracerebral hemorrhage remains a matter of debate. Though many clinical trials have attempted to better characterize the role of surgical evacuation in $\mathrm{ICH}$, variability in factors such as location and volume of the bleed and method of surgical intervention have long limited extrapolation to guidelines. Much less controversy surrounds the surgical management of infratentorial ICH due to proximity to the brain stem and the possibility of catastrophic injury and complications [20, 21]. For this reason, posterior fossa hematomas greater than $3 \mathrm{~cm}$ are evacuated due to the significant risk of brain stem compression and hydrocephalus [22].

Regardless of the cause of ICH, close monitoring of complications such as hypertension, hematoma expansion, perihematomal edema, seizures, intraventricular hemorrhage leading to hydrocephalus, and venous thromboembolism is vital to patient survival and prevention of functional deficits [23]. In addition to reducing intracranial pressure, surgical evacuation reduces clot volume, which contributes to both mechanical compression of the brain and neurotoxic edema [24]. Despite these perceived benefits, clinical trials prior to 2004 failed to demonstrate a clear survival difference in patients offered surgical intervention and medical treatment compared to conservative medical management. For example, a multicenter randomized controlled trial utilizing minimally invasive, stereotactic approaches with lowdose tissue plasminogen activator (tPA) for liquefaction and aspiration of clot (SICHPA trial) demonstrated effective reduction of clot size compared to conservative treatment, but no differences were found in 180-day mortality rates [25].

Five trials from 1989 to 2003 demonstrated equivocal outcomes following surgical intervention. Encouraging functional outcomes were demonstrated in a small study of stereotactic evacuation of putaminal hemorrhages in 2004 [26]. The International Surgical Trial in Intracerebral Hemorrhage (STICH) randomized over 1000 patients with spontaneous basal ganglia and/or lobar hemorrhages to surgery within 24 hours of presentation versus early conservative management with possible surgical evacuation after 24 hours in the setting of neurological deterioration to examine the efficacy of early surgical clot evacuation. Though the trial found only $26 \%$ of surgical patients had favorable outcomes at 6 months compared to $24 \%$ in the medical management group, subgroup analysis demonstrated that patients with supratentorial ICH with hematomas $1 \mathrm{~cm}$ or less from the cortical surface had improved outcomes with surgical evacuation compared with patients with deep hematomas and conservative management [27].

To follow up on this finding, over 600 patients with 10-100 mL superficial lobar hemorrhages and no intraventricular hemorrhage (IVH) were randomized to evacuation within 12 hours plus medical treatment compared to medical management alone with the option for subsequent surgical intervention for neurological deterioration in the STICH II trial in 2013 [28]. This trial demonstrated similarly statistically insignificant findings. Mortality at 6 months was $18 \%$ in the early surgery group compared to $24 \%$ in the medical management group, with an absolute difference of $5.6 \%$. Though the surgery group demonstrated no vegetative survivors through 6 months and the distribution of Extended Glasgow Outcome Scale (GOS-E) scores was more favorable in the surgery group, neither of these findings was statistically 
significant. However, subgroup analysis of patients with poor prognosis before treatment defined as GCS 9-12 demonstrated more favorable outcome with surgery (odds ratio of poor outcome: $0.49,95 \%$ confidence interval $0.26-0.92, p=0.02$ ). Based upon the results of the STICH II trial, the investigators concluded that patients with higher GCS of 13-15 do not demonstrate survival advantage with early surgery if given the option of delayed surgery if deterioration occurs.

The CLEAR IVH trial and MISTIE trial are ongoing investigations that use minimally invasive technique with assistance of low-dose tPA [29]. Preliminary results from the MISTIE II trial demonstrated minimally invasive aspiration with low-dose tPA reduced clots to $50 \%$ of the stabilized volume within the first week, compared to a $6 \%$ reduction with medical management alone. Though statistically significant increases in symptomatic bleeding were not seen with the use of tPA $(2.4 \%$ in the minimally invasive plus tPA group versus $9.3 \%$ in the medical management group), the authors did caution that the use of minimally invasive techniques with tPA did increase asymptomatic hemorrhages $(22.2 \%$ versus $7.1 \%, p=0.051)$. These results demonstrate the safety and efficacy of these interventions compared with conservative management alone [30]. Furthermore, hematoma evacuation has been shown to significantly reduce perihematomal edema, even when combined with tPA delivered to the clot [31]. The MISTIE Intraoperative Stereotactic Computed Tomography-Guided Endoscopic Surgery (MISTIE ICES) trial [32] was recently completed and demonstrated $42.9 \%$ of surgical patients had functional neurological outcomes defined as a modified Rankin scale score (mRS) of $0-3$, compared to $23.7 \%$ in the medical management group at 180 and 365 days (p $=0.19$ ). These results demonstrate the safety and efficacy of CT-guided endoscopic surgery to remove acute ICH. Examples of endoscopic hemorrhage evacuation can be found below (Figures 1-3).

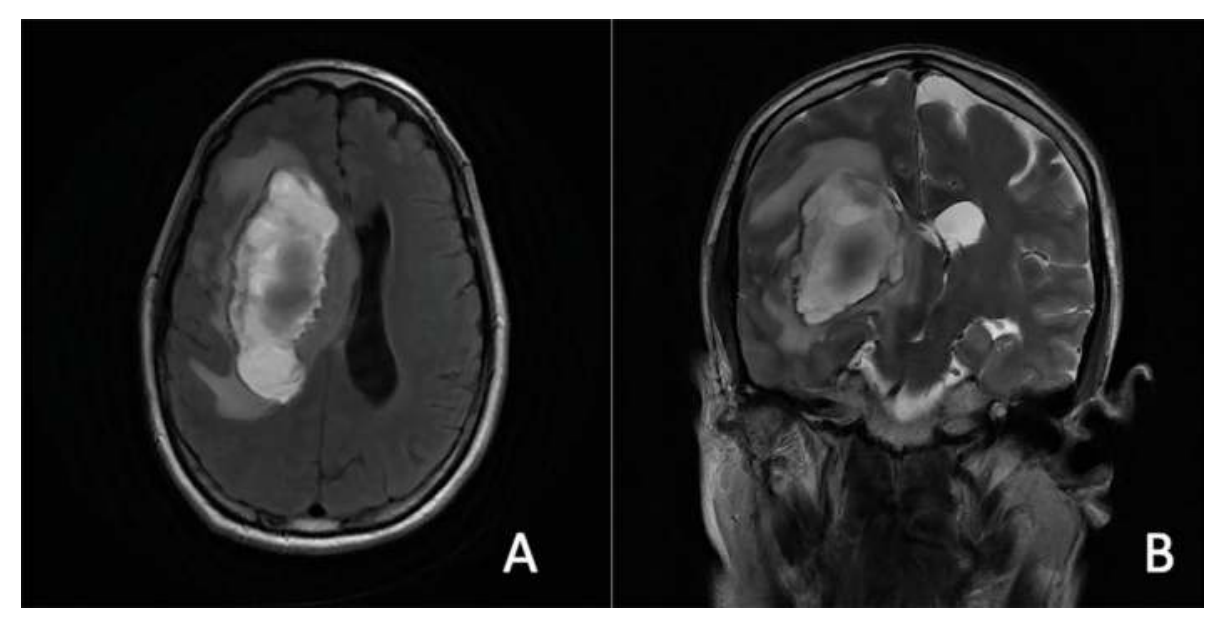

Figure 1. (A and B): Axial and coronal MRI demonstrating right-sided preoperative intracerebral hemorrhage involving the right ventricle. 


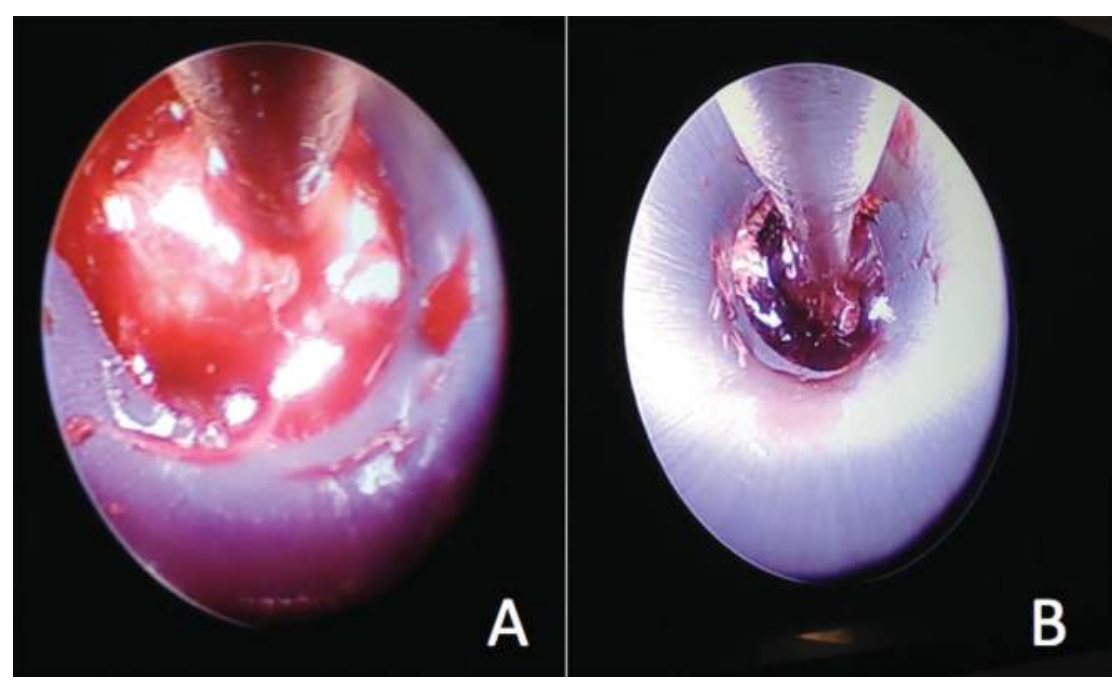

Figure 2. (A and B): Intraoperative endoscopic clot evacuation using minimally invasive technique.

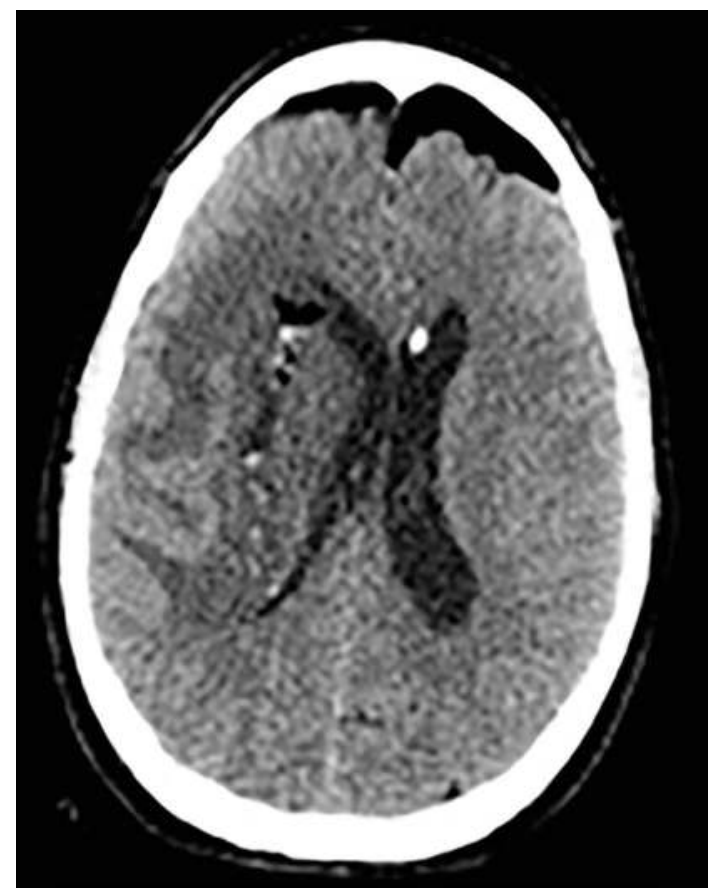

Figure 3. Follow-up axial CT scan after endoscopic hemorrhagic evacuation demonstrating resolution of clot. 


\section{Endoscopic hemorrhage evacuation example}

Taken together, the results of these trials demonstrate the difficulties in producing guidelines for the surgical management of ICH (Figures 1-3). Though surgical intervention demonstrates better outcomes in early trials, these findings do not reach statistical significance. Like many neurosurgical studies, many of these trials suffer from significant patient crossover and highly variable patient characteristics. It has been argued that the subjectively better outcomes in early trials are of clinical relevance, especially when patients are projected to have poor outcomes as a result of the location or volume of their bleed. More recent trials have demonstrated the safety and efficacy of endoscopic measures combined with low-dose tPA in dissolving clots, reducing edema, and improving outcomes to a statistically significant degree. Endoscopic approaches to ICH will likely become more widely utilized as more data from clinical trials becomes available.

\section{Postoperative management}

Postoperative management of ICH includes ensuring appropriate blood pressure control, frequent neurologic examinations, deep vein thrombosis prophylaxis, and gastric ulcer prophylaxis. Subsequent physical therapy and rehabilitation especially in the first month after ICH has been shown to be more effective in increasing independence with activities of daily life and motor function when compared to controls [33].

Despite limited evidence of long-term postoperative management of $\mathrm{ICH}$, studies suggest that $\mathrm{ICH}$ patients would benefit from a systolic blood pressure of $<130 \mathrm{mmHg}$. Patients, notably, with established small vessel disease see a $60 \%$ risk reduction in recurrent $\mathrm{ICH}$ with these blood pressure guidelines [9]. Furthermore, studies have shown that in patients with poor clinical grade or coexisting cardiopulmonary complications, early hemodynamic stabilization is associated with lower rates of delayed cerebral ischemia, lower 90-day mRS, and lower length of intensive care unit stay [34].

Deep vein thrombosis (DVT) prophylaxis after ICH is a current area of uncertainty. Various studies, including the CLOTS3 trial, show reduction in asymptomatic DVT with the use of intermittent pneumatic compression devices in ICH patients. Similarly, low molecular weight heparin or unfractionated heparin can be used for DVT prophylaxis in patients with stable hematomas or 24 hours after craniotomy [35]. There is little current evidence to suggest positive or negative outcomes of mortality with gastric ulcer prophylaxis for ICH patients. A randomized controlled trial comparing ranitidine, sucralfate, and placebo for gastric hemorrhage prophylaxis in ICH patients showed no significant difference in mortality or pneumonia. However, given the increased prevalence of gastric ulcers in these patients, prophylaxis should be initiated based on current data [36].

\section{Conclusion}

Intracerebral hemorrhage remains a serious complication associated with head trauma and a consequence of hemorrhagic stroke. Appropriate diagnosis and management of intracerebral 
pressures and ventilation preoperatively remains an important opportunity to improve patient outcomes. While surgical intervention for large infratentorial ICH is clearly beneficial, the role of supratentorial ICH remains controversial given the diversity of $\mathrm{ICH}$ locations, depth of bleed, and technique used to evacuate the hemorrhagic clot. Superficial cortical ICH can have improved outcomes with surgical evacuation compared to medical management alone, and image-guided endoscopic evacuation of clot also shows promise. ICH patients can have a host of complications in the postoperative window and require close follow-up to prevent subsequent surgical and medical complications. Further prospective trials elucidating whether surgical intervention compared to medical management alone is optimal given an ICH location, presentation, or volume of hemorrhage will continue to guide the management of this diverse population.

\section{Author details}

Arvin R. Wali, Kevin Porras, Peter Abraham, Michael G. Brandel, David Santiago Dieppa, Jeffrey Steinberg, Scott Pannell and Alexander A. Khalessi*

*Address all correspondence to: akhalessi@ucsd.edu

Department of Neurosurgery, University of California, San Diego, La Jolla, California, USA

\section{References}

[1] Rincon F, Mayer SA. The epidemiology of intracerebral hemorrhage in the United States from 1979 to 2008. Neurocrit Care 2013;19(1):95-102.

[2] Sahni R, Weinberger J. Management of intracerebral hemorrhage. Vasc Health Risk Manag 2007;3(5):701-709.

[3] Perel P, Roberts I, Bouamra O, Woodford M, Mooney J, Lecky F. Intracranial bleeding in patients with traumatic brain injury: a prognostic study. BMC Emerg Med 2009;9:15.

[4] Greenberg MS Handbook of Neurosurgery. 8th edition. ed. New York: Thieme; 2016.

[5] Broderick JP, Adams HP, Jr., Barsan W, et al. Guidelines for the management of spontaneous intracerebral hemorrhage: A statement for healthcare professionals from a special writing group of the stroke council, American heart association. Stroke 1999;30(4):905-915.

[6] Rathore SS, Hinn AR, Cooper LS, Tyroler HA, Rosamond WD. Characterization of incident stroke signs and symptoms: Findings from the atherosclerosis risk in communities study. Stroke 2002;33(11):2718-2721.

[7] Dandapani BK, Suzuki S, Kelley RE, Reyes-Iglesias Y, Duncan RC. Relation between blood pressure and outcome in intracerebral hemorrhage. Stroke 1995;26(1):21-24.

[8] Brott T, Broderick J, Kothari R, et al. Early hemorrhage growth in patients with intracerebral hemorrhage. Stroke 1997;28(1):1-5. 
[9] Hemphill JC, 3rd, Greenberg SM, Anderson CS, et al. Guidelines for the management of spontaneous intracerebral hemorrhage: A guideline for healthcare professionals from the American heart association/American stroke association. Stroke 2015;46(7):2032-2060.

[10] Vespa PM, O'Phelan K, Shah M, et al. Acute seizures after intracerebral hemorrhage: A factor in progressive midline shift and outcome. Neurology 2003;60(9):1441-1446.

[11] Bladin CF, Alexandrov AV, Bellavance A, et al. Seizures after stroke: A prospective multicenter study. Arch Neurol 2000;57(11):1617-1622.

[12] Radberg JA, Olsson JE, Radberg CT. Prognostic parameters in spontaneous intracerebral hematomas with special reference to anticoagulant treatment. Stroke 1991;22(5):571-576.

[13] Fiebach JB, Schellinger PD, Gass A, et al. Stroke magnetic resonance imaging is accurate in hyperacute intracerebral hemorrhage: A multicenter study on the validity of stroke imaging. Stroke 2004;35(2):502-506.

[14] Kidwell CS, Saver JL, Villablanca JP, et al. Magnetic resonance imaging detection of microbleeds before thrombolysis: An emerging application. Stroke 2002;33(1):95-98.

[15] Nentwich LM, Veloz W. Neuroimaging in acute stroke. Emerg Med Clin North Am 2012;30(3):659-680.

[16] Goldstein JN, Gilson AJ. Critical care management of acute intracerebral hemorrhage. Curr Treat Options Neurol 2011;13(2):204-216.

[17] Mirsen T. Acute treatment of hypertensive intracerebral hemorrhage. Curr Treat Options Neurol 2010;12(6):504-517.

[18] Dey M, Jaffe J, Stadnik A, Awad IA. External ventricular drainage for intraventricular hemorrhage. Curr Neurol Neurosci Rep 2012;12(1):24-33.

[19] Lovasik BP, McCracken DJ, McCracken CE, et al. The effect of external ventricular drain use in intracerebral hemorrhage. World Neurosurg 2016;94:309-318.

[20] Poon MT, Fonville AF, Al-Shahi Salman R. Long-term prognosis after intracerebral haemorrhage: Systematic review and meta-analysis. J Neurol Neurosurg Psychiatry 2014;85(6):660-667.

[21] Hemphill JC, 3rd, Bonovich DC, Besmertis L, Manley GT, Johnston SC. The ICH score: A simple, reliable grading scale for intracerebral hemorrhage. Stroke 2001;32(4):891-897.

[22] Morgenstern L, Hemphill 3rd J, Anderson C, et al. American Heart Association Stroke Council and Council on Cardiovascular Nursing. Guidelines for the management of spontaneous intracerebral hemorrhage: a guideline for healthcare professionals from the American Heart Association/American Stroke Association. Stroke; a journal of cerebral circulation. 2010;41(9):2108-2129.

[23] Rennert RC, Signorelli JW, Abraham P, Pannell JS, Khalessi AA. Minimally invasive treatment of intracerebral hemorrhage. Expert Rev Neurother 2015;15(8):919-933.

[24] RF, et al. Role of blood clot formation on early edema development after experimental intracerebral hemorrhage. Stroke; a journal of cerebral circulation. 1998;29(12):2580-2586. 
[25] Teernstra OP, Evers SM, Lodder J, Leffers P, Franke CL, Blaauw G. Stereotactic treatment of intracerebral hematoma by means of a plasminogen activator: A multicenter randomized controlled trial (SICHPA). Stroke 2003;34(4):968-974.

[26] Hattori N, Katayama Y, Maya Y, Gatherer A. Impact of stereotactic hematoma evacuation on activities of daily living during the chronic period following spontaneous putaminal hemorrhage: a randomized study. J Neurosurg. 2004;101(3):417-420.

[27] Mendelow AD, Gregson BA, Fernandes HM, et al. Early surgery versus initial conservative treatment in patients with spontaneous supratentorial intracerebral haematomas in the international surgical trial in intracerebral haemorrhage (STICH): A randomised trial. Lancet 2005;365(9457):387-397.

[28] Mendelow AD, Gregson BA, Rowan EN, Murray GD, Gholkar A, Mitchell PM. Early surgery versus initial conservative treatment in patients with spontaneous supratentorial lobar intracerebral haematomas (STICH II): A randomised trial. Lancet 2013;382(9890):397-408.

[29] Dey M, Stadnik A, Awad IA. Spontaneous intracerebral and intraventricular hemorrhage: advances in minimally invasive surgery and thrombolytic evacuation, and lessons learned in recent trials. Neurosurgery 2014;74(Suppl 1):S142-S150.

[30] Hanley DF, Thompson RE, Muschelli J, et al. Safety and efficacy of minimally invasive surgery plus alteplase in intracerebral haemorrhage evacuation (MISTIE): A randomised, controlled, open-label, phase 2 trial. Lancet Neurol 2016;15(12):1228-1237.

[31] Mould WA, Carhuapoma JR, Muschelli J, et al. Minimally invasive surgery plus recombinant tissue-type plasminogen activator for intracerebral hemorrhage evacuation decreases perihematomal edema. Stroke 2013;44(3):627-634.

[32] Vespa P, Hanley D, Betz J, et al. ICES (intraoperative stereotactic computed tomographyguided endoscopic surgery) for brain hemorrhage: A multicenter randomized controlled trial. Stroke 2016;47(11):2749-2755.

[33] Bai $Y, \mathrm{Hu} Y, \mathrm{Wu} Y$, et al. A prospective, randomized, single-blinded trial on the effect of early rehabilitation on daily activities and motor function of patients with hemorrhagic stroke. J Clin Neurosci 2012;19(10):1376-1379.

[34] Mutoh T, Kazumata K, Terasaka S, Taki Y, Suzuki A, Ishikawa T. Early intensive versus minimally invasive approach to postoperative hemodynamic management after subarachnoid hemorrhage. Stroke 2014;45(5):1280-1284.

[35] Nyquist P, Bautista C, Jichici D, et al. Prophylaxis of venous thrombosis in neurocritical care patients: An evidence-based guideline: A statement for healthcare professionals from the neurocritical care society. Neurocrit Care 2016;24(1):47-60.

[36] Misra UK, Kalita J, Pandey S, Mandal SK, Srivastava M. A randomized placebo controlled trial of ranitidine versus sucralfate in patients with spontaneous intracerebral hemorrhage for prevention of gastric hemorrhage. J Neurol Sci 2005;239(1):5-10. 
